\title{
Nearing final design of the ITER EC H\&CD Upper Launcher
}

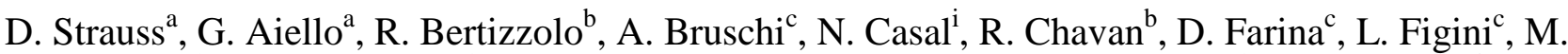 \\ Gagliardi $^{\text {h }}$, T. P. Goodman ${ }^{\mathrm{b}}$, G. Grossetti ${ }^{\mathrm{a}}$, C. Heemskerk ${ }^{\mathrm{g}}$, M.A. Henderson ${ }^{\mathrm{i}}$, W. Kasparek ${ }^{\mathrm{f}}$, J. \\ Koning $^{\mathrm{g}}$, J.-D. Landis ${ }^{\mathrm{b}}$, D. Leichtle ${ }^{\mathrm{a}}$, A. Meier ${ }^{\mathrm{a}}$, A. Moro ${ }^{\mathrm{c}}$, S. Novak ${ }^{\mathrm{c}}$, J. Pacheco ${ }^{\mathrm{h}}$, P. Platania ${ }^{\mathrm{c}}$, B. \\ Plaum $^{\text {f, E. Poli }}{ }^{\mathrm{e}}$ F. Ramseyer ${ }^{\mathrm{b}}$, D. Ronden ${ }^{\mathrm{d}}$, G. Saibene ${ }^{\mathrm{h}}$, A. Más-Sanchez ${ }^{\mathrm{b}}$, P. Santos Silva ${ }^{\mathrm{b}}$, O.

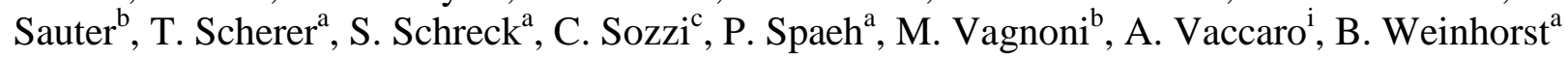 \\ ${ }^{a}$ Karlsruhe Institute of Technology, D-76021 Karlsruhe, Germany \\ ${ }^{b}$ Ecole Polytechnique Fédérale de Lausanne (EPFL), Swiss Plasma Center (SPC), CH-1015 Lausanne, Switzerland \\ ${ }^{c}$ Istituto di Fisica del Plasma CNR, 20125 Milano, Italy \\ ${ }^{d}$ DIFFER, De Zaale 20, 5612 AJ Eindhoven, Netherlands \\ ${ }^{e}$ Max-Planck-IPP, D-85748 Garching, Germany \\ ${ }^{f}$ IGVP, Pfaffenwaldring 31, D-70563 Stuttgart, Germany \\ ${ }^{g}$ Heemskerk Innovative Technology, Merelhof 2, 2172 HZ, Sassenheim, Netherlands \\ ${ }^{h}$ Fusion for Energy, Barcelona, Spain \\ ${ }^{i}$ ITER Organization, Route de Vinon sur Verdon, 13067 St Paul Lez Durance, France
}

\begin{abstract}
The ITER ECRH system consists of 24 gyrotrons with up to $24 \mathrm{MW}$ millimeter wave heating power at 170GHz, power supplies, control system, transmission lines, one Equatorial and the four Upper Launchers. With its high frequency and small beam focus the ECRH has the unique capability of driving locally current. While the Equatorial Launcher mainly acts for central heating and current profile shaping, the Upper Launchers aim on suppressing MHD instabilities, especially neoclassical tearing modes triggering plasma disruptions. The Upper Launchers inject millimeter waves through a quasi-optical section consisting of three fixed and the front steering mirror set. The eight overlapping beams have focal points optimized for suppression of the $q=3 / 2$ and $q=2 / 1$ NTMs.
\end{abstract}

Several project change requests required the redesign of the Upper Launchers and the connected ex-vessel system. This redesign includes a new boundary geometry of the launchers as well as a newly designed cooling system for the Blanket Shield Module (BSM), a modified flange of the BSM to the structural main frame and a refined optical design. Additionally shield blocks with integrated in-vessel waveguides were added and the closure plate with waveguide and supply line feedthroughs was adapted. Further changes, not all caused by PCRs, include newly designed ex-vessel waveguide components with a reduced aperture and redesigned ultra low-loss CVD diamond windows. Finally several components originally foreseen as off-the-shelf components have become part of the design scope. The new launcher design status is presented with selected results on numerical design validation.

\section{Keywords: ITER, Upper Launcher, Electron Cyclotron Heating, ECRH, Gyrotron, Plasma Heating}

\section{Introduction}

The ECRH heating system in ITER consists of 24 gyrotrons (24MW, $170 \mathrm{GHz})$, a transmission line system and the in-vessel antennas in form of one Equatorial Launcher mainly for central heating and four Upper Launchers (ULs) for plasma stabilization.

For the design of the Upper Launchers the European consortium ECHUL-CA (KIT Germany, CNR Italy, CRPP - now SPC Switzerland, IPP Garching Germany and FOM - now DIFFER Netherlands) was founded after the successful preliminary design review in 2009 [1]. The UL design (Fig. 1) is based on a fixed frequency $(170 \mathrm{GHz})$ millimeter $(\mathrm{mm})$ wave injection with angular aiming at the resonance in the plasma by friction free front steering mirrors. The main function of the UL is to stabilize the neoclassical tearing modes (NTM) for $q=3 / 2$ and $q=2 / 1$, where the assessment of stabilization criteria defines a narrow peaked density profile at the island position and a conservative minimum power deposition, both mainly depending on $\mathrm{q}$ and the toroidal injection angle $\beta$.

In late 2011 the consortium progressed with the final design [2]; several interface changes - mainly between 2013 and 2016 - required a redesign [3] of the launchers, one key issue was the reduced geometrical boundary of the ULs. In this time also an ex-vessel component design package - being part of the first confinement system [4] was added. Numerous components of the UL are SIC-1 and VQC1-A. Further the waveguide aperture was reduced from $63.5 \mathrm{~mm}$ to $50 \mathrm{~mm}$.

The beam lines pass through the UL closure plate to a quasi-optical system; the focusing and the front steering mirror units in the Blanket Shield module direct the mm-waves through a First Wall Panel (FWP) cut out into the plasma. The structural components consist of a flange connected to the VV port extension, an actively cooled socket, single and double wall main frame, shielding and the bolted Blanket Shield Module (BSM) with the FWP. 


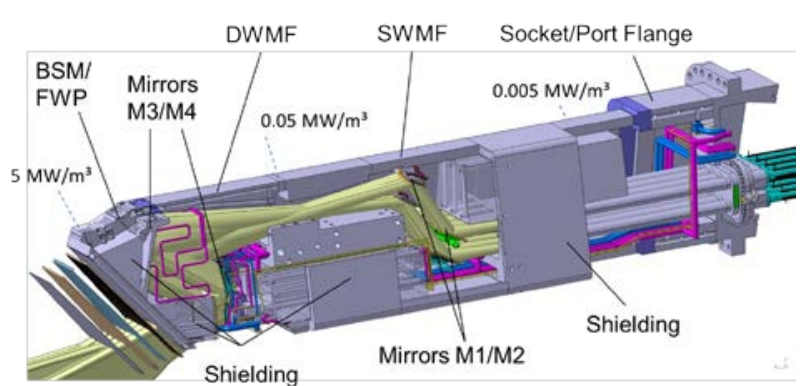

Fig. 1: The ITER ERCR Upper Launcher.

\section{Structural System}

The EC Upper Launcher is designed to the special needs of the mm-wave system requiring a wide open volume in the blanket region the EC Heating and Current Drive System. Further design drivers are maintenance [5] and manufacturing costs, here prototyping activities [6] in the past could be used to optimize the design. A new prototyping iteration [7] is currently ongoing integrating the latest design changes with a focus on BSM and FWP.

Project change requests led to a reduction of the geometrical envelope and a redesign of the structural components with the aim to conserve the volume for the optical system. The FWP consists of a steel back plate with integrated cooling lines and a plasma facing layer formed by CuCrZr with embedded cooling steel pipes. In the BSM the wall thicknesses were reduced and the cooling system [8] redesigned. As a result, several cooling lines formerly integrated into the structural components had to be replaced by free standing cooling pipes. The cut out for the mm-wave beams was adapted to the optimized optical system and the bolted flange to the launcher main frame was redesigned.

The UL Main Frame features a trapezoidal hollow structure with an actively cooled part in the front (Double Wall Main Frame), an un-cooled part (Single Wall Main Frame) as mid-section and a back part with an almost rectangular cross-section at the end. This back part is composed by a socket with embedded water channels which supports baking and the port flange with the closure plate. The port flange is part of the First Confinement System (FCS) and serves as fastener of the entire UL as well as for Vacuum and Tritium barrier.

The EC Upper launcher features also five dedicated shield blocks designed following the ALARA principle to minimize neutron flux [9]. Two of them are located in the BSM (one in the top area and one at the bottom) and three bigger ones inside the Main Frame, three further shielding zones are integrated into the wall structure.

The EC UL is designed to sustain substantial thermal and mechanical loads. The thermal loads consist of fast neutrons and photons from the plasma as well as from millimeter wave stray radiation and the associated ohmic losses. The volumetric heat load from neutrons and photons decays form ca. $5 \mathrm{MW} / \mathrm{m}^{3}$ at the very front of the port plug down to $0.005 \mathrm{MW} / \mathrm{m}^{3}$ at the back end.
The EC UL structure requires cooling from the PHTS Blanket cooling circuit. For each UL a total mass flow of $8.4 \mathrm{~kg} / \mathrm{s}$ PHTS cooling water will be provided from the ITER cooling plant. Beside the $4.5 \mathrm{~kg} / \mathrm{s}$ for the structural components, also $2.4 \mathrm{~kg} / \mathrm{s}$ water is required to cool the In-Vessel mm-wave system. A margin of $1.5 \mathrm{~kg} / \mathrm{s}$ cooling water can be used in case of additional heating. Due to maximum pressure drop of $1.35 \mathrm{MPa}$ and dedicated mass-flow requirements, the mm-wave IVcomponents require individual cooling loops, while the structural components mainly can be cooled in series. However many of the individual components require also parallel flow inside of massive structures, adjusted by orifices or static throttles. The temperature rise of the PHTS cooling water is $43 \mathrm{~K}$ while the pressure drop is calculated to be in the order of 1.0 MPa.

Mechanical loads are created mainly from Lorenz forces induced by plasma disruptions. The UL is designed to withstand the superposition of stresses from EM-loads, temperature gradients inside the port plug structure and stresses applied from deadweight.

\section{Optical System}

The in-vessel (IV) mm-wave optical components include both waveguides and metal mirrors. Waveguides pass through the closure plate and the back-end of the UL; these are arranged in two horizontal rows of 4 waveguides each. The waveguides are grouped together and cooled with water from the PHTS. They remain with the UL during any intervention requiring the transfer of the UL to the hot cell; but, they can be removed from the UL because they are bolted and sealed to the "inner" sub-plate of the PP closure plate. The ex-vessel (EX) waveguides (WGs) are thermally isolated from the IV WGs by an "outer" sub-plate, a thick block of stainless steel with 8 , corrugated, waveguide holes machined into it and cooled by water of the CCWS. The CCWS combined with the low thermal conductivity of stainless steel ensures a decrease of the temperature from the closure plate $\left(240^{\circ} \mathrm{C}\right.$ during baking) to the Aluminium waveguides, remaining below the limit of $80^{\circ} \mathrm{C}$ at which the waveguide strength starts to decrease [10].

The HE11waveguide mode couples efficiently (98\%) to a free-space Gaussian beam. Four quasi-optical (QO) mirrors reflect each beam through the UL. The 1st [11] and 3rd mirrors along the beam path are focusing (quadratic curvatures), the 2nd [12] and 4th are flat. At the 3rd mirror (M3), two reflecting surfaces are used one for each row of beams - since the beams of the row overlap: both M3 mirrors are held by the same frame; all other mirrors have individual frames (supports) per row. The 4th mirrors (M4s) steer the beams of their row to aim at desired locations in the plasma.

Each beam can be characterized by a beam "size" transverse to the direction of propagation; and each mirror size is designed to be at least a factor $\mathrm{S}$ larger than the beam size. Mirrors M1 and M2 have S=2, M3 and M4 have $\mathrm{S}=1.5$. For a pure Gaussian beam this would correspond to capturing $99.966 \%$ and $98.889 \%$ of 
the power in the beam, respectively: the missing power is diffracted around the edges of the mirror. Since the beams do not overlap at the M1 and M2 positions, each beam is reflected off of its own mirror; and though the 4 mirrors of each row are on the same frame, the 4 are segmented from each other to allow for independent thermal expansion of each reflecting surface, minimizing distortions. This segmentation is not possible on mirrors M3 and M4, but it is done on M3 between rows.

The curvatures of the M1 surface focus the beam equally in the tangential and sagittal planes, but the M3 curvatures are optimized to focus the beams of each row in the plasma to maintain as tight an overlap as possible at the intersection between the $\mathrm{q}=2$ and resonance surfaces for the ITER $Q=10, I_{p}=15$ MA scenario. As the beams are steered away from this location, the overlap decreases, so the narrowness and peak intensity of the driven current channel also decreases.

Subsequent to the design optimization, other input conditions are analyzed with respect to the beam propagation in the launcher using the commercial code GRASP [13] and in the plasma using GRAY [14]; that is, an HE11 input at the end of each IV WG is propagated (as opposed to a Gaussian beam) through the system and the reflecting surfaces are distorted according to the thermo-mechanical analysis results. This provides localized first-pass stray radiation incident powers on the UL structure and an estimation of the deviation of the beams from their optimal (design) paths in the launcher. At the opposite extreme, stray radiation is treated with an "integrating-sphere" model (random, geometric-series-like, multiple reflections) to provide an "average" thermal load on different sections of the launcher (M1/M2 region, M3/M4 region, and the passage between them) under different surface conditions (copper-coated, or bare stainless steel). Stray radiation absorption intensities are used as input thermal loads on the structures, ranging from $14 \mathrm{~kW} / \mathrm{m}^{2}$ (copper coated surface) to $95 \mathrm{~kW} / \mathrm{m}^{2}$ (stainless steel) locally in the worst location; average loads range between $10 \mathrm{~kW} / \mathrm{m}^{2}$ (fully copper-coated surfaces throughout the launcher) to $48 \mathrm{~kW} / \mathrm{m}^{2}$ (stainless-steel)

The deviations of the beams are used to quantify loss of NTM stabilization efficiency. In addition to beam deviations due to distortions, for 5\% wrong mode content significant deviations of the beam axis can occur. For a mixture of the two lowest order modes in the waveguide this can lead to most-likely-estimates of randomly distributed offsets of $2.5 \mathrm{~mm}$ and angles of $0.28^{\circ}$. Random orientations of the deviations lead to suggested distances to be kept between the beams and the mechanical structures: these are incorporated into the design. In addition, the sensitivity of the usual output angles $(\alpha, \beta)$ to errors in the input deviations is calculated and these errors are then translated into errors in the radial deposition location $(\rho)$ in the plasma as a function of the input deviations.

\section{Ex-vessel System}

The UL is connected to the transmission line system via 8 beamlines passing through the closure plate, two sets of miter bends provide flexibility compensating e.g. thermal expansion and a ceiling support structure protects mechanically isolation valves and diamond windows [15]. The beamlines are connected to the dcbreak and the transmission line system. The design is ongoing and in the following the torus diamond windows will be described in more detail.

The diamond window is designed for $1.31 \mathrm{MW}$ incident beam power. As it further acts as vacuum and tritium confinement barrier, the window unit has stringent requirements in the ITER safety, quality, seismic, vacuum and tritium classifications [16].

The window unit consists of a $1.11 \mathrm{~mm}$ thick, $80 \mathrm{~mm}$ diameter ultra-low loss chemical vapor deposition (CVD) diamond disk brazed to two copper cuffs and then welded into a CuCrZr-housing. The rigid outer window frame protects the diamond disk from external loads. With indirect cooling there is no direct contact between disk and coolant. Corrugated waveguides are inserted into the cuffs leaving a $100 \mu \mathrm{m}$ gap with the diamond disk to prevent parasitic electromagnetic oscillations in the small cavities of the unit.

The selected code for the design, manufacturing, assembly and qualification of the unit is ASME Sec. III - Subsection NC. Further an ad-hoc qualification program is required as the brazing between disk and copper cuffs is not covered by standard codes [17]. The diamond disk ITER Final Design Review is in the process of being closed. The window design has been advanced by FEM analyses [18] and the application of the ASME code to start soon the manufacturing of prototypes in order to successfully pass the FDR of the window unit, scheduled at the end of 2019.

\section{Performance}

The main physics objective driving the design of the UL is control and suppression of Neoclassical Tearing Modes (NTMs), although a number of other applications are foreseen [19]. To best achieve this aim, the EC current should be localized within a region corresponding to the radial extension of the NTM at its marginal size, which is still not predicted by the theory but is assumed to be in the range of a few (1-6) $\mathrm{cm}$. The present design ensures small beam spot size, of the order of $3 \mathrm{~cm}$, at the positions of interest, namely the magnetic surfaces characterized by the values $q=2$ and $q=3 / 2$ of the safety factor. As a reference for this and the following estimates, the plasma parameters predicted for the nuclear burn phase of the standard $Q=10$-scenario have been taken, although an extensive analysis has been performed to include the whole time evolution of this and other scenarios [20]. The toroidal injection angle is fixed to $20^{\circ}$, leading to the maximum EC current compatible with the radial localization mentioned above. For a single beam, beam tracing calculations predict an EC deposition profile below $4 \mathrm{~cm}$ at the $\mathrm{q}=2$-surface and below $5 \mathrm{~cm}$ at the $\mathrm{q}=3 / 2$-surface. The power needed for 
NTM suppression, estimated through simplified criteria and verified by a more detailed analysis involving a numerical solution of Rutherford equation [21 and references therein], is found to be of the order of $7 \mathrm{MW}$, which can be delivered by continuous injection. Also at different times and for different plasma scenarios the available power should be sufficient to achieve NTM control. The design is being finalized in order to obtain the best overlap of the four beams injected from each mirror compatible with the engineering constraints. Several further effects which could lead to broader EC profiles than predicted by a standard beam tracing analysis have been studied. Beam scattering from turbulent density fluctuations, located mainly at the plasma edge, is believed to be the most deleterious and has been addressed in detail [22]. In the most likely regime of parameters a broadening by a factor of 2.5 to 3.5 is found, reducing the NTM stabilization efficiency. For marginal NTM widths of the order of 1-2 cm, this implies that power modulation in-phase with the O-point of the NTM might become necessary.

\section{Outlook}

As the Project Change Requests implied a substantial redesign and thereby delay $\mathrm{IO}$ and F4E decided to prepone the manufacturing design phase to the preparation of the Final Design Review. The benefit is, that in parallel to the last design steps the preparation of a design ready for procurement can be started accelerating the progress towards manufacturing with additional support from industry. As the design contract between ECHUL-CA and F4E ends in 11/2018 the hand over to F4E has been initiated, starting with the structural design. Some design work on critical details is still ongoing as the mitigation of e.g. hot spots at the launcher cut out by adding an actively cooled CuCrZr layer similar to the First Wall Panel as well as shielding optimization. Individual optical components including diamond windows will be further developed by research centres being part of ECHUL-CA. The Final Design Reviews for the ITER ECRH Upper Launchers will continue in 2019 with the In-Vessel components.

\section{Acknowledgments}

This work was supported by Fusion for Energy under the grant contract No. F4E-2010-GRT-161 and by ITA C52TD39FE. The views and opinions expressed herein reflect only the author's views. Fusion for Energy and ITER are not liable for any use that may be made of the information contained therein.

\section{References}

[1] D. Strauss et al., Preliminary design of the ITER ECH Upper Launcher, Fusion engineering and Design 88 (2013) 2761-2764.

[2] D. Strauss et al., Progress of the ECRH Upper Launcher design for ITER, Fusion Engineering and Design 89
(2014) 1669-1673.

[3] P. Spaeh et al., Status of the Final design of the EC UPP Launcher, Fusion Engineering and Design, 123 (2017) 341-344

[4] A. Mas Sánchez, et al., Mechanical analyses of the ITER electron cyclotron upper launcher first confinement system, Fusion Eng. Des. 123 (2017) 458-462

[5] D.M.S. Ronden et al., The ITER EC H\&CD Upper Launcher: Maintenance concepts, Fusion Engineering and Design 88 (2013)

[6] P. Spaeh et al., Prototyping of the Blanket Shield Module for the ITER EC H\&CD Upper launcher, Fusion Engineering and Design, 89 (2014) 960-964

[7] J. Pacheco et al., Cooling optimization of the electron cyclotron upper launcher blanket shield module, SOFT 2018

[8] P. Spaeh et al., Update of the cooling design of the ITER EC Upper Launcher, Proceedings of the 29th Symposium On Fusion Technology (SOFT-29), Prague, Czech republic, 05 - 09 Sep. 2016

[9] P. Spaeh et al., Design of the ITER EC Upper Launcher Nuclear Shielding, SOFT 2018

[10] A. Mas Sánchez, et al., Design status of the double Closure Plate Sub-Plate concept for the ITER Electron Cyclotron Upper Launcher, Fusion Eng. Des. In Press

[11] M. Vagnoni, et al., Thermo-mechanical analysis of an ITER ECH\&CD Upper Launcher mirror, Fusion Eng. Des. In Press, Corrected Proof (2018)

[12] P. Santos Silva et al., Design Concept and ThermalStructural Analysis of a High Power Reflective mm-wave Optical Mirror (M2) for the ITER ECH-UL, SOFT 2018

[13] K. Pontoppidan, GRASP Technical Description, TICRA, Denmark (2005)

[14] D. Farina, A Quasi-Optical Beam-Tracing Code for Electron Cyclotron Absorption and Current Drive: GRAY, Fusion Science and Technology 52 (2007) 154160

[15] T. A. Scherer et al., Investigations of microwave and THz radiation losses in CVD diamond and chemically modified diamond, MRS Fall Meeting (2010), Invited talk, Proceedings Symp. A, Vol. 1282, pp. 177 -186S.

[16] Schreck et al., Pressure tests supporting the qualification of the ITER EC H\&CD upper launcher diamond window, SOFT 2018

[17] S. Schreck et al., ITER ECRH upper launcher: test plan for qualification of the diamond torus window prototype III, Fusion Engineering and Design 109-111 (2016) 1232-1236

[18] G. Aiello et al., The ITER EC H\&CD upper launcher: methodology in the FEM analyses of the diamond window unit subject to seismic and baking loads, Proceedings of the 26th Symposium on Fusion Engineering, 31 May - 4 June 2015, Austin, TX, USA, 2015 IEEE, ISBN 978-1-4799-8264-6, ISSN 2155-9953.

[19] Henderson et al., Phys. Plasmas 22, 021808 (2015)

[20] Figini et al., Plasma Phys. Control. Fusion 57, 054015 (2015)

[21] Poli et al., Nucl. Fusion 55, 013023 (2015)

[22] Snicker et al., Nucl. Fusion 58, 016002 (2018) 